\title{
Política de saúde LGBT e sua invisibilidade nas publicações em saúde coletiva
}

\author{
LGBT health policy and its invisibility in public health publications
}

Marcos Vinicius da Rocha Bezerra', Camila Amaral Moreno', Nília Maria de Brito Lima Prado', Adriano Maia dos Santos ${ }^{1}$

DOI: $10.1590 / 0103-11042019 S 822$

RESUMO Trata-se de uma revisão de literatura que tem por objetivo identificar o que expressaram as publicações dos principais periódicos nacionais da área da saúde coletiva, sobre gestão, políticas e práticas em saúde direcionadas à população LGBT, no período compreendido entre 2004 e 2018. A busca de documentos se deu por meio da ferramenta 'busca integrada', sendo selecionados artigos disponíveis na íntegra que estavam indexados na base de dados Scientific Electronic Library Online (SciELO). Foram analisadas as publicações de dez periódicos nacionais, e selecionadas 27 publicações que discutiam políticas públicas de saúde para a população LGBT. Observou-se pouca expressão de temas relacionados com a população LGBT, nos 14 anos do recorte temporal proposto por este artigo, uma vez que, dos 14.700 artigos encontrados, somente 92 faziam referência à população LGBT, e apenas 27 discutiam as políticas de saúde. Desse modo, verifica-se a necessidade de realização de pesquisas direcionadas à análise de implantação das políticas públicas de saúde LGBT, que subsidiem a construção de dados epidemiológicos e informações que traduzam as necessidades em saúde dessa população.

PALAVRAS-CHAVE Políticas de saúde. Minorias sexuais e de gênero. Equidade de gênero.

ABSTRACT This is a literature review that aims to identify what is expressed in the publications of the main nationals journals regarding management, policies, and health practices addressed to the LGBT population, in the period comprehended between 2004 and 2018. The search of documents was carried out through the tool 'integrated search', being selected available articles in their whole that were indexed in the database Scientific Electronic Library Online (SciELO). We analyzed the publications of 10 national periodics and selected 27 publications that addressed health public policies towards the LGBT population. Little expression of related themes regarding the LGBT population was observed, in the 14 years of the temporary cut proposed by this article, since from the articles found, only 92 made reference the LGBT population and only 27 discussed health policies. Thus, it is verified the need for accomplishment of researches addressing the analysis of implantation of health public policies towards LGBT, that subsidize the construction of epidemiological data and information that translate the needs in the health of that population.

KEYWORDS Health policy. Sexual and gender minorities. Gender equity. 


\section{Introdução}

A partir da década de 1970, intensificaram-se, em países latino-americanos, os movimentos em prol das reformas no setor social, com o intuito de minimizar as desigualdades socioeconômicas e reduzir a pobreza da população, bem como reestruturar ou fortalecer os sistemas de saúde'. No Brasil, as bases do movimento da Reforma Sanitária Brasileira (RSB) inscritas na Constituição Federal de 1988, e regulamentadas pelas Leis $\mathrm{n}^{\circ} 8.080 / 90$ e $\mathrm{n}^{\mathrm{o}} 8.142 / 90$, alicerçaram o caminho para a construção de uma política universal e integral, sinalizando a necessidade de inclusão de populações até então marginalizadas.

O processo de redemocratização da saúde, a partir do caráter contra hegemônico da RSB, propiciou a abertura para o debate sobre cidadania e direitos sociais que reverbera em importantes contribuições para a construção de um modelo de assistência pautado na equidade em saúde ${ }^{2}$.

Nesse contexto político, emergiram diversos grupos sociais, como os de Lésbicas, Gays, Bissexuais, Travestis e Transsexuais (LGBT) que passaram a se articular por maior visibilidade, melhorias na qualidade de vida, igualdade de gênero e orientação sexual e pela luta por direitos civis ${ }^{3}$, bem como para a resolução de questões inconclusas referentes à epidemia de Vírus da Imunodeficiência Humana/Síndrome da Imunodeficiência Adquirida (HIV/Aids) ${ }^{4}$.

No campo da saúde coletiva, surgem, após a criação do Sistema Único de Saúde (SUS), as políticas de promoção da equidade, que têm por objetivo diminuir as vulnerabilidades a que certos grupos populacionais estão expostos, intervindo nos determinantes sociais da saúde. Ancoradas no princípio da justiça social, as políticas de equidade em saúde passaram a constituir uma das bandeiras dos movimentos sociais, em particular, o LGBT.

Compreendendo a questão saúde como objeto de ação política, diversos movimentos sociais organizados apresentaram demandas e especificidades aos governantes tendo em vista que, embora a RSB tenha propiciado a permeabilidade para as demandas das minorias sociais, tais diálogos obtiveram uma penetração heterogênea no âmbito governamental. Esse fato corroborou o desenvolvimento tardio de políticas públicas de saúde, como observado para população LGBT, impulsionadas somente a partir de 2004 com a publicação do Plano Brasil Sem Homofobia.

Esse marco jurídico constituiu uma ampla estratégia para ampliação, reconhecimento e fortalecimento da cidadania e dos direitos humanos no Brasil e significou não apenas a legitimidade das demandas LGBT, mas também a institucionalização e maior participação do movimento social nos espaços de elaboração de políticas de saúde em todos os âmbitos federativos ${ }^{5}$. Do Plano Brasil Sem Homofobia, nasceram ramificações nos setores do Estado, a exemplo de coordenações, planos, políticas e programas ${ }^{6-9}$.

Posto isso, pôde-se observar as particularidades, complexidade e a garantia de políticas públicas que perpassam a questão dos direitos LGBT. Entre estes, o reconhecimento da orientação sexual e identidade de gênero como determinante social da saúde'; o direito ao uso do nome social ${ }^{6,10}$; e o acesso ao Processo Transexualizador ( $\mathrm{PrTr}$ ) no serviço público de saúde ${ }^{4,11}$, que se constituíram estratégias para ampliar o acesso da população LGBT aos serviços de saúde.

Embora sejam inegáveis os progressos na formulação de políticas de saúde, em particular as relacionadas com o combate à homofobia e de promoção da cidadania e dos direitos humanos da população LGBT, ao que parece, não se logrou proporcionar a equidade e a igualdade no atendimento a essa população no SUS ${ }^{12}$, constituindo um desencontro aos princípios defendidos pela RSB.

Para melhor compreender essa hipótese, faz-se necessário identificar o que expressaram as publicações dos principais periódicos nacionais da área da saúde coletiva sobre as políticas em saúde direcionadas à população LGBT, no período compreendido entre 2004 e 2018. 


\section{Aspectos metodológicos}

Trata-se de síntese integrativa da produção científica veiculada pelos principais periódicos nacionais da área da saúde coletiva. O recorte temporal parte da publicação do Plano Brasil Sem Homofobia, em 2004, por ser um marco no desenvolvimento de políticas públicas específicas a essa população.

Os dez periódicos eleitos estão indexados na Scientific Electronic Library Online (SciELO), quais sejam: 'Cadernos de Saúde Coletiva'; 'Cadernos de Saúde Pública'; 'Ciência e Saúde Coletiva'; 'Revista Brasileira de Epidemiologia'; 'Revista Interface: comunicação, saúde, educação'; 'Revista de Saúde Pública'; 'Revista Saúde e Sociedade'; 'Saúde em Debate'; 'Revista Physis' e 'Revista Trabalho, Educação e Saúde’. A busca das revistas se deu por meio da ferramenta 'busca integrada', sendo selecionados artigos disponíveis na íntegra.

Os descritores foram identificados nos Descritores em Ciências da Saúde (DeCS), em idiomas português (Minorias Sexuais e de Gênero), inglês (Sexual and Gender Minorities) e espanhol (Minorías Sexuales y de Género). As estratégias de busca incluíram sinônimos dos descritores: Pessoas LGBT; Minorias de Gênero; Pessoas LGBTQ; Mulheres Lésbicas; Pessoa Lésbica; Pessoas LBG; Pessoas Lesbigays; Pessoas não Heterossexuais. Também foram incluídos os descritores transexuais e travestis, uma vez que estes não estavam contemplados nas categorias abordadas pelo descritor Minorias Sexuais e de Gênero. Foram eleitos artigos nos idiomas inglês, espanhol e português.
Os critérios de inclusão dos artigos foram: 1) artigos disponíveis na íntegra; 2) possuir relação com a política pública de saúde LGBT no período 2004 a 2018; 3) publicados em periódicos brasileiros na área da saúde coletiva, classificados com qualis A1 a B2, excetuando-se a revista 'Saúde em Debate' e os 'Cadernos de Saúde Coletiva', cujos artigos publicados estavam disponíveis on-line somente a partir do ano de 2012. Como critério de exclusão: artigos com acesso restrito; periódicos com qualis menor que B2 e fora do período delimitado; assuntos divergentes da temática central deste estudo. A coleta de dados ocorreu em março de 2019.

A seleção e a extração dos dados dos documentos foi realizada em quatro etapas: $1^{\mathrm{a}}$ etapa: busca e seleção dos artigos; $2^{\mathrm{a}}$ etapa: leitura de todos os títulos e resumos; $3^{\text {a }}$ etapa: leitura na íntegra e sistematização dos artigos que apresentavam publicações relacionadas a temática central; e $4^{\mathrm{a}}$ etapa: identificação e classificação dos artigos selecionados, de acordo com a área de concentração da saúde coletiva epidemiologia; política, planejamento e gestão em saúde; e ciências humanas e sociais.

Na etapa inicial da revisão, foram identificados 92 artigos. Na sequência, foram excluídos 65 artigos após a leitura de títulos e resumos, sendo que 57 não debatiam a temática central (implícita e/ou explicitamente), ou se referiam à identidade de gênero e/ou fatores de risco para transmissão do vírus HIV/Aids, sem abordar aspectos relacionados com o ciclo da política pública de saúde LGBT, e ainda 8 que não retratavam a experiência brasileira. Ao final, foram selecionados 27 artigos (figura 1). 
Figura 1. Fluxograma de busca e estratificação de artigos

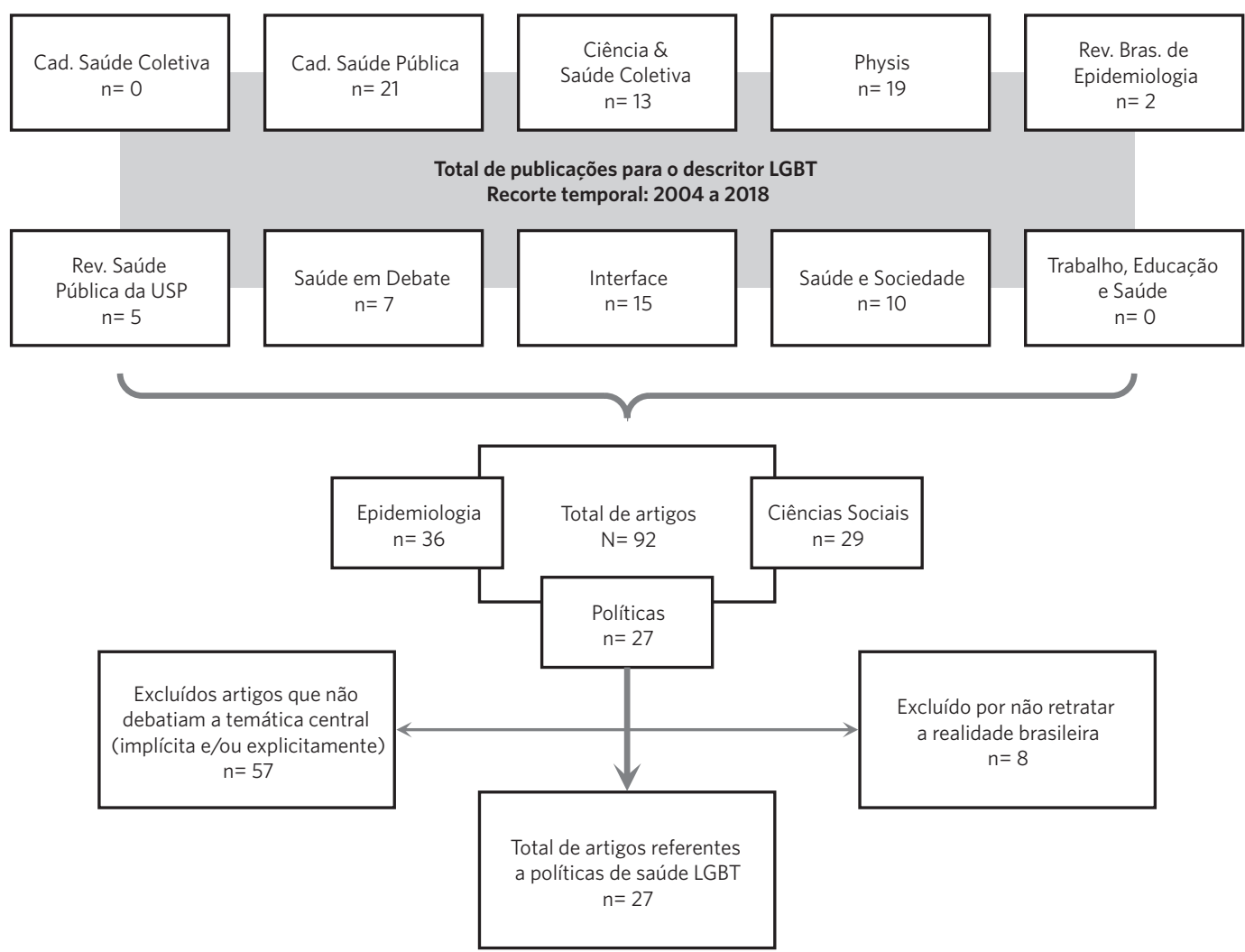

Fonte: Elaboração própria.

Cada artigo foi analisado segundo os critérios: ano de publicação, periódico, metodologia, área da saúde coletiva, aspectos relacionados com as políticas de saúde LGBT e principais desafios. Após a finalização da matriz de resultados, foi realizada uma comparação quantitativa das publicações disponíveis entre os periódicos, para balanceamento de publicações relacionadas com a política de saúde LGBT (tabela 1).

Os resultados foram apresentados segundo dois tópicos, quais sejam: 1. Caracterização geral dos artigos; 2. Perspectivas históricas das políticas de saúde LGBT. Ademais, a discussão, para ampliar a compreensão contemplou três subtópicos, conforme as três grandes áreas do campo da saúde coletiva: 1 . Área de epidemiologia: vulnerabilidade e afastamento de pessoas LGBT do cuidado em saúde; 2 . Área de ciências sociais: heteronormatividade e padrão heterossexual compulsório nos serviços de saúde; e, 3. Área de políticas, planejamento e gestão em saúde: dimensões de acesso à atenção à saúde. 
Tabela 1. Número de publicações nos periódicos de saúde coletiva

\begin{tabular}{lrrr}
\hline Revistas/Periódicos & $\begin{array}{r}\text { Total de } \\
\text { Publicações }\end{array}$ & $\begin{array}{r}\text { Total de publicações } \\
\text { LGBT }\end{array}$ & $\begin{array}{r}\text { Publicações sobre } \\
\text { Políticas LGBT }\end{array}$ \\
\hline Cadernos de Saúde Coletiva & 375 & 00 & 00 \\
Cadernos de Saúde Pública & 3.462 & 21 & 05 \\
Ciência e Saúde Coletiva & 4.152 & 13 & 04 \\
Revista Brasileira de Epidemiologia & 1.064 & 02 & 00 \\
Revista Interface & 940 & 15 & 05 \\
Revista de Saúde Pública da USP & 1.887 & 05 & 01 \\
Revista Saúde e Sociedade & 1.214 & 10 & 02 \\
Revista Physis & 762 & 19 & 06 \\
Revista Trabalho, Educação e Saúde & 466 & 00 & 00 \\
Saúde em Debate & 378 & 07 & 04 \\
\hline Total & $\mathbf{1 4 . 7 0 0}$ & $\mathbf{9 2}$ & $\mathbf{2 7}$ \\
\hline
\end{tabular}

Fonte: Elaboração própria.

\section{Resultados}

\section{Caracterização geral dos artigos}

Foram analisadas as publicações de dez periódicos nacionais veiculadas entre 2004 e 2018. Destaca-se que, nesse recorte temporal, foram publicados 14.700 artigos, sendo que, destes, somente $92(0,6 \%)$ faziam referência à população LGBT, e apenas 27 (0,2\%) discutiam políticas públicas de saúde envolvendo tal população.

Ao contrastar as publicações conforme as áreas de concentração da saúde coletiva, encontraram-se: 5 artigos na área de epidemiologia, 19 artigos na área de política, planejamento e gestão em saúde; e 3 artigos na área de ciências sociais e humanas. Entre os periódicos investigados, os 'Cadernos de Saúde Pública' apresentaram maior número de publicações referente à população LGBT (21 artigos), porém a 'Revista Physis' abordou, em maior número, as políticas públicas de saúde LGBT ou questões relacionadas (6 artigos).

Entre todas as publicações, a maioria (12) abordou a população de travestis e transexuais. Por sua vez, em relação aos temas centrais, destacaram-se: discriminação, preconceito e violência à LGBT; acesso a serviços de saúde; assistência integral à saúde da população LGBT; equidade em saúde; PrTr; vulnerabilidade da população LGBT; direitos sexuais e reprodutivos da população LGBT; e medidas de prevenção/controle de Infecções Sexualmente Transmissíveis (IST) e HIV/Aids.

A respeito da distribuição das publicações por área de concentração da saúde coletiva, constatou-se que as publicações na área da Epidemiologia abordaram a política associada ao HIV/Aids ${ }^{13,14}$; aos comportamentos de risco ${ }^{15}$; ao uso de drogas lícitas, ilícitas ${ }^{16}$ e à violência ${ }^{17}$. Na área de ciências sociais e humanas, as discussões centraram-se na vulnerabilidade à infecção por HIV e outras IST ${ }^{\mathbf{1}}$, direitos sexuais e reprodutivos da população transexual e travestis ${ }^{19} \mathrm{e}$ políticas públicas à luz da teoria queer ${ }^{20}$.

$\mathrm{Na}$ área de política, planejamento e gestão em saúde, os debates circunscreveram, em sua grande maioria, o acesso aos serviços de saúde 11,12,17,21-28, as fragilidades dos 
atendimentos em decorrência do preconceito e discriminação institucional 12,17,21,23-26,28-32, violência contra população LGBT ${ }^{29}$, tecnologias e saberes na prevenção do HIV/Aidss3,34, adolescência e homossexualidade ${ }^{35,36}$, garantia do direito à saúde 11,12,17,23-26,28,30

Quadro 1. Identificação dos títulos dos artigos publicados sobre políticas de saúde LGBT nos principais periódicos da saúde coletiva, por área de concentração, 2004-2018

\begin{tabular}{|c|c|c|c|c|}
\hline Autor/ano/periódico & Título & Metodologia & $\begin{array}{l}\text { Aspectos relacionados a } \\
\text { políticas LGBT }\end{array}$ & Desafios \\
\hline \multicolumn{5}{|c|}{ ÁREA DA SAÚDE COLETIVA: EPIDEMIOLOGIA } \\
\hline $\begin{array}{l}\text { Pinto TP, Teixeira FB, } \\
\text { Barros CRS, et al. Cad. } \\
\text { Saúde Pública. } 2017 .\end{array}$ & $\begin{array}{l}\text { Silicone líquido industrial para } \\
\text { transformar o corpo: preva- } \\
\text { lência e fatores associados } \\
\text { ao seu uso entre travestis e } \\
\text { mulheres transexuais em São } \\
\text { Paulo, Brasil. }\end{array}$ & $\begin{array}{l}\text { Estudo Transver- } \\
\text { sal/576 travestis } \\
\text { e mulheres } \\
\text { transexuais. }\end{array}$ & $\begin{array}{l}\text { Processo Transexua- } \\
\text { lizador para acesso a } \\
\text { práticas de modificações } \\
\text { corporais/ uso de Sili- } \\
\text { cone Líquido Industrial } \\
\text { (SLI). }\end{array}$ & $\begin{array}{l}\text { Presença de obstáculos estruturais no acesso } \\
\text { e oferta de procedimentos no SUS; idade } \\
\text { mínima para uso de silicone industrial e para o } \\
\text { processo transexualizador. }\end{array}$ \\
\hline $\begin{array}{l}\text { Brignol S, Dourado } \\
\text { I, Amorim LD, et al. } \\
\text { Cad. Saúde Pública. } \\
2014 .\end{array}$ & $\begin{array}{l}\text { Vulnerabilidade no contexto } \\
\text { da infecção por HIV e sífilis } \\
\text { numa população de Homens } \\
\text { que fazem Sexo com Homens } \\
\text { (HSH) no Município de Sal- } \\
\text { vador, Bahia, Brasil. }\end{array}$ & $\begin{array}{l}\text { Estudo transver- } \\
\text { sal, técnica de } \\
\text { recrutamento } \\
\text { RDS/383 HSH. }\end{array}$ & $\begin{array}{l}\text { A vulnerabilidade social, } \\
\text { individual e programática } \\
\text { dos HSH, no contexto do } \\
\text { HIV/DSTs. }\end{array}$ & $\begin{array}{l}\text { Redução da vulnerabilidade e do estigma } \\
\text { de populações e indivíduos frente ao HIV/ } \\
\text { AIDS e DSTs; acesso oportuno aos serviços e } \\
\text { informações de saúde sobre risco de infecção, } \\
\text { diagnóstico e tratamento do HIV/DST. }\end{array}$ \\
\hline $\begin{array}{l}\text { Magno L, Dourado } \\
\text { I, Silva LA, et al. Rev } \\
\text { Saude Publica. } 2017 .\end{array}$ & $\begin{array}{l}\text { Fatores associados à discri- } \\
\text { minação autorreferida contra } \\
\text { homens que fazem sexo com } \\
\text { homens no Brasil. }\end{array}$ & $\begin{array}{l}\text { Estudo transver- } \\
\text { sal/3.859 HSH. }\end{array}$ & $\begin{array}{l}\text { A vertente das barreiras } \\
\text { ao acesso dos serviços } \\
\text { de saúde, a busca pela } \\
\text { realização teste anti HIV, } \\
\text { discriminação pelo gene- } \\
\text { ro e identidade sexual. }\end{array}$ & $\begin{array}{l}\text { Discriminação potencial dos serviços sobre o } \\
\text { aconselhamento para DSTs frente à orienta- } \\
\text { ção sexual de HSH. }\end{array}$ \\
\hline $\begin{array}{l}\text { Albuquerque GA, } \\
\text { Parente JS, Belém JM, } \\
\text { et al. Rev. Saúde em } \\
\text { Debate. } 2016 .\end{array}$ & $\begin{array}{l}\text { Violência psicológica em } \\
\text { lésbicas, gays, bissexuais, } \\
\text { travestis e transexuais no } \\
\text { interior do Ceará, Brasil. }\end{array}$ & $\begin{array}{l}\text { Estudo transver- } \\
\text { sal/316 LGBTT. }\end{array}$ & $\begin{array}{l}\text { Combate à violência } \\
\text { psicológica/verbal contra } \\
\text { a população LGBTT. }\end{array}$ & $\begin{array}{l}\text { Combater o preconceito, a hostilidade, a natu- } \\
\text { ralização dos atos de violência, omissão social } \\
\text { permissiva direcionados a LGBTT. }\end{array}$ \\
\hline $\begin{array}{l}\text { Rocha RMG, Pereira } \\
\text { DL, Dias TM. Rev. } \\
\text { Saúde e Sociedade. } \\
2013 .\end{array}$ & $\begin{array}{l}\text { O contexto do uso de drogas } \\
\text { entre travestis profissionais } \\
\text { do sexo. }\end{array}$ & $\begin{array}{l}\text { Estudo descriti- } \\
\text { vo/ } 59 \text { travestis } \\
\text { profissionais do } \\
\text { sexo (prontuá- } \\
\text { rios). }\end{array}$ & $\begin{array}{l}\text { Uso de drogas nos espa- } \\
\text { ços de prostituição po- } \\
\text { tencializa a vulnerabilida- } \\
\text { de no contexto de vida e } \\
\text { trabalho das travestis. }\end{array}$ & $\begin{array}{l}\text { Combater o preconceito e discriminação; } \\
\text { Fortalecer a rede de proteção as travestis } \\
\text { profissionais do sexo e a e a necessidade de } \\
\text { desenvolver estratégias e ações para redução } \\
\text { de danos. }\end{array}$ \\
\hline \multicolumn{5}{|c|}{ ÁREA DA SAÚDE COLETIVA: CIÊNCIAS SOCIAIS E HUMANAS } \\
\hline $\begin{array}{l}\text { Almeida. Physis: Rev. } \\
\text { Saúde Coletiva. } 2009 .\end{array}$ & $\begin{array}{l}\text { Argumentos em torno da } \\
\text { possibilidade de infecção por } \\
\text { DST e Aids entre mulheres } \\
\text { que se autodefinem como } \\
\text { lésbicas. }\end{array}$ & $\begin{array}{l}\text { Estudo qualitati- } \\
\text { vo/ } 10 \text { mulheres } \\
\text { lésbicas e gine- } \\
\text { cologistas. }\end{array}$ & $\begin{array}{l}\text { Vulnerabilidade indi- } \\
\text { vidual, programática } \\
\text { e social da população } \\
\text { lésbica; invisibilidade das } \\
\text { questões relacionadas a } \\
\text { orientação sexual, para } \\
\text { infecção pelo HIV/DST; } \\
\text { movimento social na } \\
\text { articulação e efetivação } \\
\text { de ações voltadas a } \\
\text { população lésbica. }\end{array}$ & $\begin{array}{l}\text { Superar práticas preconceituosas a população } \\
\text { lésbica nos serviços de saúde; } \\
\text { Promover mudanças na formação dos profis- } \\
\text { sionais de saúde para lidar com as questões } \\
\text { relacionadas a pluralidade de gênero e orien- } \\
\text { tação sexual; } \\
\text { Ampliação de ações de divulgação de infor- } \\
\text { mações junto ao coletivo lésbico referentes a } \\
\text { práticas sexuais; } \\
\text { Mudança na cultura sexual das mulheres } \\
\text { lésbicas. }\end{array}$ \\
\hline
\end{tabular}


Quadro 1. (cont.)

\begin{tabular}{|c|c|c|c|c|}
\hline Autor/ano/periódico & Título & Metodologia & $\begin{array}{l}\text { Aspectos relacionados a } \\
\text { políticas LGBT }\end{array}$ & Desafios \\
\hline $\begin{array}{l}\text { Motta JIJ. Rev. Saúde } \\
\text { em Debate. } 2016 .\end{array}$ & $\begin{array}{l}\text { Sexualidades e políticas } \\
\text { públicas: uma abordagem } \\
\text { queer para tempos de crise } \\
\text { democrática. }\end{array}$ & $\begin{array}{l}\text { Ensaio Teórico/ } \\
\text { LGBT }\end{array}$ & $\begin{array}{l}\text { Categorias poder, resis- } \\
\text { tência e transgressão } \\
\text { à luz da teoria queer, } \\
\text { no contexto da Política } \\
\text { Nacional de Saúde Inte- } \\
\text { gral LGBT. }\end{array}$ & $\begin{array}{l}\text { Efetivar o princípio da integralidade do cui- } \\
\text { dado frente a um modelo de assistência as- } \\
\text { sentado na heteronormatividade e no padrão } \\
\text { heterossexual compulsório; } \\
\text { Efetivar o controle social da população LGBT } \\
\text { nos espaços de produção das políticas rela- } \\
\text { cionadas a diversidade de gênero e orientação } \\
\text { sexual. }\end{array}$ \\
\hline $\begin{array}{l}\text { Angonese M, Lago } \\
\text { MCS. Rev. Saúde e } \\
\text { sociedade. } 2017 .\end{array}$ & $\begin{array}{l}\text { Direitos e saúde reprodutiva } \\
\text { para a população de traves- } \\
\text { tis e transexuais: abjeção e } \\
\text { esterilidade simbólica. }\end{array}$ & $\begin{array}{l}\text { Estudo etnográfi- } \\
\text { co/ } 4 \text { travestis e } \\
\text { transexuais. }\end{array}$ & $\begin{array}{l}\text { Invisibilidade dos direitos } \\
\text { sexuais e reprodutivos } \\
\text { para travestis e transe- } \\
\text { xuais ante ao modelo } \\
\text { heterocisnormativo } \\
\text { como uma barreira para } \\
\text { a saúde sexual e repro- } \\
\text { dutiva. }\end{array}$ & $\begin{array}{l}\text { Promover mudanças na formação dos pro- } \\
\text { fissionais de saúde preparando-os para atuar } \\
\text { frente a diversidade de gênero e orientação } \\
\text { sexual; } \\
\text { Adequar os serviços de saúde para o atendi- } \\
\text { mento à população trans; } \\
\text { Desconstruir a noção heterocisnormativa que } \\
\text { tem orientado os serviços e profissionais de } \\
\text { saúde no atendimento a travestis e transe- } \\
\text { xuais. }\end{array}$ \\
\hline
\end{tabular}

\section{ÁREA DA SAÚDE COLETIVA: POLÍTICAS, GESTÃO, PLANEJAMENTO E AVALIAC̄̃̃O}

Barbosa RM, Facchini Acesso a cuidados relativos à R. Cad. Saúde Pública. saúde sexual entre mulheres 2009 que fazem sexo com mulheres em São Paulo, Brasil.
Redoschi BRL, Zucchi EM, Barros CRS, et al. Cad. Saúde Pública. 2017.

Sousa D, Iriart J. Cad. Saúde Pública. 2018.

Silva CG, Paiva V Parker R. Interface Comunicação, Saúde, Educação. 2013.
Uso rotineiro do teste anti-HIV entre homens que fazem sexo com homens: do risco à prevenção.

'Viver dignamente': necessidades e demandas de saúde de homens trans em Salvador, trans. Bahia, Brasil.

Juventude religiosa e homossexualidade: desafios para a promoção da saúde e de direitos sexuais
Estudo qualitativo / 30 mulheres que fazem sexo com mulheres.
Revisão narrativa Estratégias programátida literatura/ $\mathrm{HSH}$.

Estudo qualitativo/ 10 homens cas para enfrentamento do HIV/AIDS previstas na Meta 90-90-90, proposta pelo UNAIDS até 2030.

Despatologização, a modificação corporal e
Convenções sociais e de gênero para o acesso a cuidados ginecológicos não associados a heterossexualidade ou a maternidade. para erradicar a epidemia os atendimentos ambulatoriais e reconhecimento das necessidades e demandas de saúde dos homens trans.

Estudo qualita- Concepções de jovens tivo/18 jovens religiosos sobre homosreligiosos de 15 a sexualidade relevantes 25 anos .
Qualificar os profissionais de saúde para o atendimento às necessidades de saúde dessa população;

A menor procura de mulheres que fazem sexo com mulheres em decorrência da discriminação;

Dificuldade das mulheres em revelar a orientação sexual

Ampliar o teste anti-HIV como uma estratégia preventiva pelos atores envolvidos na produção das políticas públicas

Ausência de pesquisas e indicadores epidemiológicos que expressem quadros de saúde dos homens trans;

Presença de transfobia nos serviços de saúde; Ausência do processo transexualizador e mercantilização das demandas de saúde quanto as modificações corporais

Ampliação do diálogo dos formuladores de políticas públicas de saúde com os jovens religiosos e as autoridades religiosas estabelecendo a distinção entre dogma e religiosidade; públicas para promoção da saúde sexual na cultura dominada pela tradição judaico-cristã. 
Quadro 1. (cont.)

\begin{tabular}{|c|c|c|c|c|}
\hline Autor/ano/periódico & Título & Metodologia & $\begin{array}{l}\text { Aspectos relacionados a } \\
\text { políticas LGBT }\end{array}$ & Desafios \\
\hline $\begin{array}{l}\text { Taquette SR, Rodri- } \\
\text { gues AO. Interface - } \\
\text { Comunicação, Saúde, } \\
\text { Educação. } 2015 .\end{array}$ & $\begin{array}{l}\text { Experiências homossexuais } \\
\text { de adolescentes: considera- } \\
\text { ções para o atendimento em } \\
\text { saúde. }\end{array}$ & $\begin{array}{l}\text { Estudo qua- } \\
\text { litativo/ } 14 \\
\text { adolescentes } \\
\text { de } 12 \text { a } 19 \text { anos } \\
\text { com experiência } \\
\text { homossexual. }\end{array}$ & $\begin{array}{l}\text { Significados das práticas } \\
\text { homoafetivas de adoles- } \\
\text { centes em contextos de } \\
\text { vulnerabilidade. }\end{array}$ & $\begin{array}{l}\text { Considerar a orientação sexual dos adoles- } \\
\text { centes nos serviços de saúde. }\end{array}$ \\
\hline $\begin{array}{l}\text { Rocon PC, Sodré } \\
\text { F, Zamboni J, et al. } \\
\text { Interface - Comunica- } \\
\text { ção, Saúde, Educação. } \\
2018 .\end{array}$ & $\begin{array}{l}\text { O que esperam pessoas trans } \\
\text { do Sistema Único de Saúde? }\end{array}$ & $\begin{array}{l}\text { Estudo qualita- } \\
\text { tivo/15 pessoas } \\
\text { trans }\end{array}$ & $\begin{array}{l}\text { Garantia de acesso e da } \\
\text { promoção de ações para } \\
\text { cuidado integral a pes- } \\
\text { soas trans no SUS. }\end{array}$ & $\begin{array}{l}\text { Necessidade de formação continuada para } \\
\text { trabalhadores da saúde para reverter a reali- } \\
\text { dade de desrespeito do nome social; } \\
\text { Universalizar a hormonioterapia, o implante } \\
\text { de silicone e o atendimento multiprofissional; } \\
\text { Obrigatoriedade do diagnóstico mediador do } \\
\text { acesso aos serviços transgenitalizadores. }\end{array}$ \\
\hline
\end{tabular}

\section{ÁREA DA SAÚDE COLETIVA: POLÍTICAS, GESTÃO, PLANEJAMENTO E AVALIAC̣ÃO}

Valadão RC, Gomes

R. Physis: Rev. Saúde
Coletiva. 2011
Estudo qualita-

lésbicas e bissexuais.
tivo/Mulheres
Lionço T. Physis: Rev. Saúde Coletiva. 2009.
Atenção integral à saúde e

diversidade sexual no Processo Transexualizador do SUS: avanços, impasses, desafios.
Ensaio teórico/

Travestis e transexuais.
Crítica aos modelos mativos que provocam a invisibilidade, discriminação e preconceito da homossexualidade/ lesbiandade na política integral de saúde da mulher.

Ambivalência na construção da norma técnica do PrTr no SUS, discutindo a judicialização para regulamentação e financiamento dos procedimentos médico-cirúrgicos.

Ensaio teóri- Utilização do nome soco-reflexivo/ cial como condição funTravestis e tran- damental para promover o acesso e acolhimento de travestis e transexuais nos serviços de saúde.

Ferreira BO, Nascimento EF, Pedrosa JIS, et al. Physis: Rev. Saúde Coletiva. 2017.
Uso do nome social no Sistema Único de Saúde: elementos para o debate sobre a assistência prestada a sexuais. travestis e transexuais.

Vivências de travestis no acesso ao SUS.
Estudo qualitativo/ 6 travestis.
Ramos S, Carrara S. Physis: Rev. Saúde Coletiva. 2006.
A Constituição da Problemática da Violência contra Homossexuais: a Articulação entre Ativismo e Academia na Elaboração de Políticas Públicas. biomédicos e heteronor-

Combater o preconceito, discriminacão e invisibilidade acerca da lesbiandade. Necessidade de formação continuada para trabalhadores da saúde para adoção de boas práticas em saúde voltadas para a diversidade da sexualidade e identidade de gênero.

Obrigatoriedade da condição diagnóstica (transexualismo) para acesso ao PrTr; Democratizar os direitos sexuais, numa perspectiva de diversidade sexual.

Preconceito e discriminação nos serviços de saúde;

Capacitar os profissionais de saúde para lidar coma as questões relacionadas a identidade sexual e de gênero.

Discriminação e preconceito nos serviços públicos de saúde;

Proporcionar acolhimento humanizado; Ampliar o acesso aos serviços de saúde reduzindo a procura por serviços precários e clandestinos tanto para a modificação corporal quanto para os cuidados em saúde.

Ensaio teórico/ Discute as diferentes for- Necessidade de criação de dispositivos e População LGBT. mas de violência sofridas articulações em diferentes setores capazes de pela população LGBT. articulações em diferentes setores capazes 
Quadro 1. (cont.)

\begin{tabular}{|c|c|c|c|c|}
\hline Autor/ano/periódico & Título & Metodologia & $\begin{array}{l}\text { Aspectos relacionados a } \\
\text { políticas LGBT }\end{array}$ & Desafios \\
\hline $\begin{array}{l}\text { Zago LF, Santos LHS. } \\
\text { Physis: Rev. Saúde } \\
\text { Coletiva. } 2013 .\end{array}$ & $\begin{array}{l}\text { Os limites do conceito de } \\
\text { empoderamento na preven- } \\
\text { ção ao HIV/Aids entre jovens } \\
\text { gays e bissexuais no Brasil }\end{array}$ & $\begin{array}{l}\text { Pesquisa qualita- } \\
\text { tiva/ Jovens gays } \\
\text { e bissexuais }\end{array}$ & $\begin{array}{l}\text { Emprego da metodolo- } \\
\text { gia do empoderamento } \\
\text { como conceito-estraté- } \\
\text { gia em uma política de } \\
\text { prevenção ao HIV/Aids. }\end{array}$ & $\begin{array}{l}\text { Adaptar o conceito de empoderamento } \\
\text { proposto para atender as dimensões sociais } \\
\text { e políticas em que se encontram inserida a } \\
\text { população de jovens gays e bissexuais }\end{array}$ \\
\hline
\end{tabular}

\section{ÁREA DA SAÚDE COLETIVA: POLÍTICAS, GESTÃO, PLANEJAMENTO E AVALIAC̣ÃO}

FREIRE et al. Rev. Saúde em Debate. 2013.

Albuquerque $\mathrm{GA}$ Garcia CL, Alves MJH, et al. Rev. Saúde em Debate. 2013.

Lionço T. Rev. Saúde e Sociedade. 2008.

Gomes SM, Sousa LMP, Vasconcelos TM, et al. Rev. Saúde e Sociedade. 2018.

Popadiuk GS, Oliveira DC, Signorelli MC. Rev. Ciências e Saúde Coletiva. 2017.

\section{A clínica em movimento na saúde de TTTS: caminho para materialização do SUS entre travestis, transsexuais e transgêneros. \\ Ensaio teórico/ travestis, trangê- neros e transe- xuais.}

Homossexualidade e o direito à saúde: um desafio para as políticas públicas de saúde no Brasil.

Que Direito à Saúde para a População GLBT? Considerando Direitos Humanos, Sexuais e Reprodutivos em Busca da Integralidade e da Eqüidade.

O SUS fora do armário: concepcões de gestores municipais de saúde sobre a população LGBT.

A Política Nacional de Saúde Integral de Lésbicas, Gays, Bissexuais e Transgêneros (LGBT) e o acesso ao Processo Transexualizador no Sistema Único de Saúde (SUS): avanços e desafios.

Revisão de literatura/ LGBT.

Ensaio teórico/ LGBT.

Estudo qualitativo/ 12 diretores e coordenadores.
Estudo quanti e qualitativo/ LGBT.

politicas públicas LGB

Criação de politicas públicas de saúde para população LGBT no âmbito do SUS.

Desconhecimento das diretrizes da PNSI-LGBT pelos gestores em saúde do município de Cuité (PB) e os seus reflexos nos serviços de saúde.

Apresenta as principais conquistas da população LGBT no campo da saúde e discute as implantação do processo transexualizador ( $\mathrm{Pr} T r)$ no SUS.
Necessidade de aç̃es específicas voltadas a população em questão dentro e fora das unidades.

Homofobia institucional;

Ausência de dotação orçamentária para as políticas LGBT

Capacitação dos profissionais acerca das demandas da diversidade sexual e de gênero; Reformulação dos espaços de saúde para atendimento humanizado a população LGBT

Discriminação e preconceito nos serviços de saúde;

Estigma institucional;

Desnaturalizar e desmedicalizar a sexualidade reconhecendo a homossexualidade como uma possibilidade de constituição humana legítima;

Democratizar os direitos sexuais reprodutivos

Desarticulação dos níveis de atenção à saúde; Desconhecimento da condição de saúde vivenciada pela população LGBT no município: Formação continuada de gestores e profissionais para o reconhecimento das especificidades e necessidades da população em questão; Discriminação e preconceito

Discriminação, o estigma e o preconceito em relação a identidade de gênero e orientação sexual;

Capacitação dos profissionais de saúde para acolhimento e ampliação do acesso da população trans;

Adequar o sistema de marcação de procedimentos exames para possibilitar a inclusão das pessoas trans;

Reduzida quantidade de hospitais e ambulatórios habilitados para realização do PrTr. 
Quadro 1. (cont.)

\begin{tabular}{|c|c|c|c|c|}
\hline Autor/ano/periódico & Título & Metodologia & $\begin{array}{l}\text { Aspectos relacionados a } \\
\text { políticas LGBT }\end{array}$ & Desafios \\
\hline \multicolumn{5}{|c|}{ ÁREA DA SAÚDE COLETIVA: POLÍTICAS, GESTÃO, PLANEJAMENTO E AVALIAC̣ÃO } \\
\hline $\begin{array}{l}\text { Souza MHT, Signorelli } \\
\text { MC, Coviello DM, } \\
\text { et al. Rev. Ciências e } \\
\text { Saúde Coletiva. } 2014 .\end{array}$ & $\begin{array}{l}\text { Itinerários terapêuticos de } \\
\text { travestis da região central do } \\
\text { Rio Grande do Sul, Brasil. }\end{array}$ & $\begin{array}{l}\text { Estudo qualitati- } \\
\text { vo/ } 49 \text { travestis. }\end{array}$ & $\begin{array}{l}\text { Itinerário terapêutico das } \\
\text { travestis de Santa Maria } \\
\text { (RS), na busca de cui- } \\
\text { dados em saúde dentro } \\
\text { de instituições não con- } \\
\text { vencionais (religião de } \\
\text { matriz africana). }\end{array}$ & $\begin{array}{l}\text { Discriminação e preconceito institucional; } \\
\text { Capacitar e qualificar os profissionais para } \\
\text { atendimento das demandas das travestis; } \\
\text { Respeito ao uso do nome social nas institui- } \\
\text { ções de saúde; } \\
\text { Ampliação do acesso à serviços de saúde. }\end{array}$ \\
\hline $\begin{array}{l}\text { Rocon PC, Rodrigues } \\
\text { A, Zamboni J, et al. } \\
\text { Rev. Ciências e Saúde } \\
\text { Coletiva. } 2016 .\end{array}$ & $\begin{array}{l}\text { Dificuldades vividas por } \\
\text { pessoas trans no acesso ao } \\
\text { Sistema Único de Saúde. }\end{array}$ & $\begin{array}{l}\text { Pesquisa qualita- } \\
\text { tiva/ } 15 \text { pessoas } \\
\text { trans. }\end{array}$ & $\begin{array}{l}\text { Dificuldades enfrenta- } \\
\text { das por pessoas trans } \\
\text { ao acesso aos serviços } \\
\text { públicos de saúde na } \\
\text { Grande Vitória (ES). }\end{array}$ & $\begin{array}{l}\text { Desrespeito ao nome social, discriminação e } \\
\text { o diagnóstico no PrTr como principais limita- } \\
\text { ções no acesso aos serviços de saúde; } \\
\text { Capacitar e qualificar os profissionais de } \\
\text { saúde para o atendimento de pessoas trans } \\
\text { nos serviços; } \\
\text { Ampliar o número de instituições habilitadas } \\
\text { a realizar o processo transexualizador no } \\
\text { SUS e ampliar o acesso de pessoas trans aos } \\
\text { serviços de saúde. }\end{array}$ \\
\hline
\end{tabular}

Fonte: Elaboração própria.

\section{Perspectivas históricas das políticas de saúde LGBT}

No final da década de 1970, no Brasil, surgiram diversos movimentos sociais em defesa dos direitos dos grupos lésbicas, gays, bissexuais, travestis e transexuais. Tais grupos travaram lutas objetivando romper com o estereótipo, bem como com as diversas formas de violência (sexual, psicológico, física e o preconceito). Sob essa ótica, o público LGBT passou a ser visualizado com um novo olhar no cenário da política de saúde; e assim surgiram discussões que até então não eram fortalecidas ou referenciadas.

Em uma perspectiva histórica, evidências científicas apontam avanços na implantação de políticas direcionadas à saúde da população LGBT. A trajetória do movimento LGBT obteve maior ascensão social a partir da redemocratização brasileira. Nesse período, iniciou-se o processo de institucionalização do movimento homossexual vinculado às ações de prevenção das Doenças Sexualmente Transmissíveis (DST), quando ocorre um fortalecimento dos movimentos sociais diante da epidemia da Aids, e a reconfiguração de muitos destes grupos sociais em Organizações Não Governamentais (ONG) ${ }^{29}$.

A década de 1990 foi marcada pelo aumento do número de organizações do movimento, pela sua diversificação de atuação e de formatos institucionais ${ }^{37}$. Nesse cenário, ampliou-se a rede de articulação do movimento com a presença de novos atores sociais e maior visibilidade da diversidade sexual como questão política e social. Entre outras iniciativas, destaca-se a primeira Parada do Orgulho LGBT, em São Paulo, pela inclusão de ativistas e homossexuais não organizados por meio de uma política de visibilidade massiva ${ }^{37}$.

A partir de 2004, foram publicados (figura 2), pelo Ministério da Saúde, diferentes planos, programas e políticas com intuito de combater a discriminação e o preconceito, promover a cidadania e ampliar o acesso da população 
LGBT aos serviços de saúde. Ainda assim, as publicações foram restritas e não refletiram em pesquisas os avanços políticos.

O Plano Brasil Sem Homofobia, lançado pelo governo federal em 2004, foi criado para a formulação de políticas e programas específicos na intenção da melhoria da situação de vida dos grupos LGBT. Em resposta aos debates e à ausculta de demandas de representações do movimento social, as diretrizes pautaram-se no intuito de promover a inserção de ações que atendessem a especificidades de saúde desse contingente populacional.

O reduzido número de publicações, mesmo diante de importantes instrumentos normativos, assevera a baixa permeabilidade de temas relacionados com LGBT nos periódicos estudados. Uma possível explicação para esse fato apoia-se na ideia de que os temas referentes a essa população não foram considerados relevantes para compor os periódicos estudados, não se alinhavam com as linhas editoriais das revistas ou não havia produção significativa de pesquisas, na saúde coletiva, que abarcasse tal população.

Por sua vez, em conjunturas favoráveis e de maior abertura ao tema e em períodos de governos progressistas e democráticos, a comunidade LGBT galgou avanços nas políticas públicas implementadas nas diversas áreas sociais, especialmente no setor saúde.

Nessa seara, em 2006, o Conselho Nacional de Saúde passou a contar em sua composição com a representação LGBT $^{\mathbf{1 0}}$, e o Ministério da Saúde lançou a 'Carta do usuário do SUS' que, entre outras ações, prevê $o$ atendimento humanizado, acolhedor e livre de qualquer discriminação a essa população, além de abrir caminho para o uso do nome social ${ }^{38}$. Tal ação influencia diretamente no acolhimento à população transgênero. Embora constitua importante conquista à cidadania de travestis e transexuais, poucas publicações analisaram as barreiras de acesso aos serviços de saúde para tal população.

Os anos de 2007 a 2011 foram marcados por eventos significativos para a garantia de direitos, promoção da cidadania e desenvolvimento de políticas públicas para população LGBT.
Não obstante tenha-se instituído o Plano Integrado de Enfrentamento da Feminização da Epidemia de Aids e DST ${ }^{7}$, o Plano Nacional de Enfrentamento da Epidemia de Aids e DST entre gays, Homens que fazem Sexo com Homens (HSH) e travestis ${ }^{39}$; o Plano Nacional de Promoção da Cidadania e Direitos Humanos de Lésbicas, Gays, Bissexuais, Travestis e Transexuais (PNPCDH-LGBT) ${ }^{\mathbf{8}}$; o $\mathrm{PrTr}^{40}$; e a Política Nacional de Saúde Integral de Lésbicas, Gays, Bissexuais, Travestis e Transexuais (PNSI-LGBT) ${ }^{9}$, houve apenas cinco publicações a respeito da implantação dessas políticas, da implementação das ações desenvolvidas e do impacto destas na saúde da população LGBT. Além disso, chama a atenção a insuficiência de estudos de avaliação das políticas LGBT para a produção de dados epidemiológicos, avanço do conhecimento e fabricação de novas tecnologias voltadas para o cuidado em saúde dessa população.

Depois de muitas versões, durante a XIV Conferência Nacional de Saúde, a PNSILGBT foi assinada em 2011, instituindo a democratização do direito à saúde a essa parcela populacional. Essa conquista teve grande implicação dos movimentos sociais em diálogo com instituições de pesquisa e ONG para que as propostas formuladas e defendidas pudessem, de fato, adentrar a agenda governamental e ser formalizada.

Desde então, a PNSI-LGBT tem-se constituído de acordo com os princípios e diretrizes do SUS, de forma universal e gratuita. Destacamos que, nos anos subsequentes, houve maior expressão no contexto nacional dos avanços relacionados com o PrTr no SUS, do acesso de travestis e transexuais aos serviços de saúde, e as conquistas civis, como o uso do nome social no SUS, a adoção de crianças por casais homoafetivos; direito ao uso do nome social; direito de homossexual receber pensão pela morte de seu cônjuge; reconhecimento da união estável de casais homoafetivos; possibilidade de constar o cônjuge homossexual na identidade militar; licença-maternidade a pai adotivo gay, e o casamento civil gay. Todavia, é importante 
destacar que todas essas conquistas precisam de maior efetivação e responsabilidade universal por parte do Estado, principalmente no que se refere ao processo de inclusão e equidade.

Em suma, as políticas públicas surgem na intenção de resguardar e proteger a integridade física, moral, mental e identitária da população LGBT, mas cabe ao Estado adotar todos os instrumentos necessários para que se assegure a estes indivíduos, historicamente marginalizados, o pleno acesso e proteção ao direito fundamental à saúde.

Figura 2. Linha do tempo dos principais marcos sociais, normativos e técnicos para o desenvolvimento de políticas LGBT no Brasil

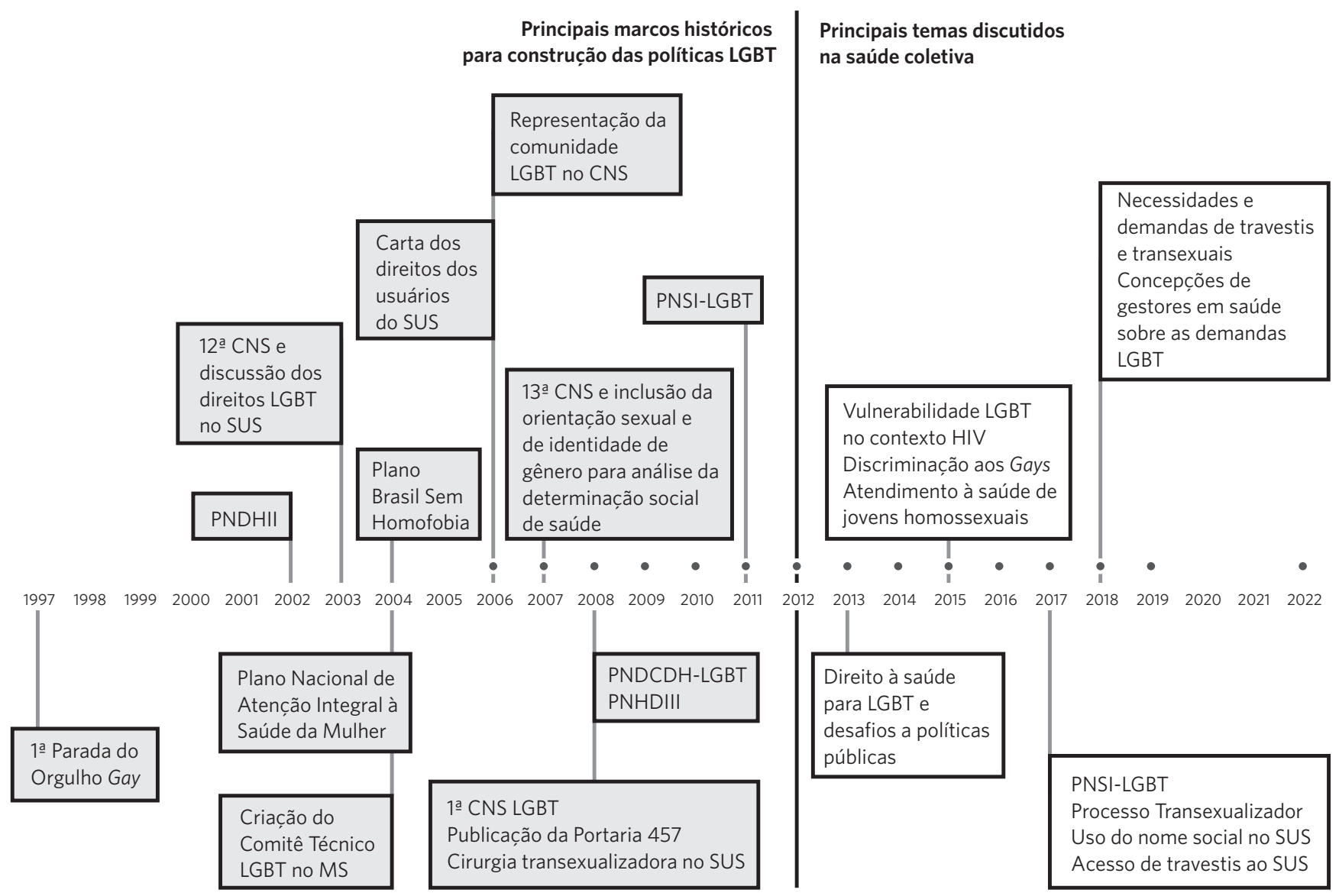

\section{Discussão}

Os temas relacionados com a população LGBT tiveram pouca expressividade no cômputo geral das publicações identificadas nos últimos
15 anos. Entretanto, os poucos artigos trouxeram importantes contribuições ao tratarem de temas complexos e apontarem vulnerabilidades em decorrência da identidade de gênero e orientação sexual. 


\section{Área de epidemiologia: vulnerabili- dade e afastamento de pessoas LGBT do cuidado em saúde}

Ao desvelar fatores associados à prática de uso de silicone industrial entre travestis e mulheres transexuais ${ }^{15}$, apontou-se a necessidade de assegurar o acesso aos recursos necessários para realização das modificações corporais ao longo do percurso de transição. Para tanto, o artigo aponta para a necessidade de políticas públicas que reconheçam a diversidade das demandas de saúde desse grupo social, na perspectiva de construção da identidade de gênero pautada na singularidade com que cada indivíduo vivencia seu processo de transição.

$\mathrm{Na}$ mesma direção, o uso e abuso de drogas por travestis profissionais do sexo que frequentavam um serviço de saúde apontou a importância de que uma política integrada seja desenvolvida para aumentar os repertórios de existência dessas pessoas, em termos de educação, saúde e trabalho, historicamente restringidos ${ }^{16}$.

Em sintonia com os demais temas, a violência psicológica praticada contra LGBT sinaliza um fenômeno complexo e multidimensional, enquanto reflexo do preconceito e hostilidade direcionados às expressões de sexualidade não heteronormativas, além de figurar como problema de saúde pública, evidenciando a necessidade primordial de conhecimento das especificidades de assistência a cada grupo da comunidade LGBT ${ }^{41}$.

Por fim, a vulnerabilidade social, individual e programática dos HSH, no contexto da epidemia do HIV/DST ${ }^{13}$, bem como a discriminação desse grupo ${ }^{14}$ demonstram a complexa cadeia relacional entre estigma e adoecimento que, por sua vez, culmina no afastamento do público LGBT do cuidado em saúde, e a necessidade da articulação de ações voltadas para promoção de ações diferenciadas, com o objetivo de reduzir desigualdades de grupos em condições de exclusão social e iniquidade em saúde, mesmo quando este é disponibilizado no SUS.
Por outro lado, uma política de enfrentamento das iniquidades da população LGBT requer iniciativas estratégicas vigorosas, entre as quais, a capacitação dos profissionais de saúde sobre as práticas sexuais e sociais de LGBT. Esta é questão fundamental para que o cuidado seja condizente com suas reais necessidades em saúde.

Em síntese, os artigos abordaram a perspectiva da integralidade da atenção à saúde desses segmentos populacionais brasileiros e o descaso dos profissionais de saúde e dos serviços, por exporem a população LGBT a agravos decorrentes do estigma, dos processos discriminatórios e de exclusão social que violam seus direitos humanos, entre os quais, o direito à saúde, à dignidade, à não discriminação, à autonomia e ao livre desenvolvimento.

\section{Área de ciências sociais: heteronor- matividade e padrão heterossexual compulsório nos serviços de saúde}

Ao discutir as possibilidades de infecção por DTS e Aids, aponta-se que práticas de saúde forjadas em padrões heteronormativos, associadas à cultura sexual de mulheres lésbicas, expõe esse grupo a diferentes tipos de vulnerabilidade (individual, programática e social) ${ }^{\mathbf{1 8}}$, culminando no afastamento dos serviços de saúde, na desinformação das mulheres lésbicas acerca das formas de infecção por DTS/Aids, ao mesmo tempo que alimentam fragilidades nos programas de saúde desenvolvidos para as mulheres. $\mathrm{O}$ autor destaca a necessidade de mudanças na formação dos profissionais de saúde, superando a etnocentricidade e a visão generalizante que levam ao despreparo técnico para lidar com as questões de orientação e práticas sexuais.

Em concordância, ao discutir a 'esterilidade simbólica' de travestis e transexuais, os autores sinalizam o modelo de atenção heterocisnormativo ${ }^{19}$. Para tal, debatem como a constituição de sujeitos abjetos está dissociada de questões como reprodução e parentalidade, mostrando que o gozo dos direitos sexuais e reprodutivos 
não é uma realidade atual, especialmente para a população trans. Ademais, apontam para reestruturação dos serviços de saúde para acolhimento das demandas e especificidades dessa população, bem como para a desconstrução da heteronormatividade como noção orientadora de práticas em saúde, além da urgência de alteração na formação acadêmica dos profissionais de saúde.

Do mesmo modo, ao estudar categorias de poder, resistência e transgressão à luz da teoria queer, no contexto da PNSI-LGBT, apontam-se os desafios de efetivar o princípio da integralidade perante um modelo de assistência assentado na heteronormatividade e no padrão heterossexual compulsório ${ }^{20}$, bem como de efetivar o controle social da população LGBT, demarcar e assegurar a sua inserção em espaços de construção de políticas públicas.

\section{Área de políticas, planejamento e gestão em saúde: dimensões de acesso à atenção à saúde}

Os artigos da área de política, planejamento e gestão em saúde convergiram para o debate acerca de distintas dimensões do acesso aos serviços e aos cuidados em saúde. Especificamente no que se refere ao acesso, vale enfatizá-lo como parte essencial para o cuidado da saúde LGBT.

Nesse sentido, é importante destacar as questões predominantes nos diversos estudos analisados, quais sejam: a inclusão e a articulação das especificidades de orientação sexual e identidade de gênero em uma política nacional voltada para as populações LGBT; a necessidade de implementação de práticas de educação permanente para os profissionais de saúde, incluindo a temática LGBT; a definição de normas não discriminatórias e respeito ao direito à intimidade e à individualidade dos grupos e indivíduos pertencentes às populações LGBT; a necessidade de garantia dos direitos sexuais e reprodutivos extensiva a esses segmentos; a implementação de campanhas e revisão dos currículos escolares; e as mudanças nos formulários, prontuários e sistemas de informação do SUS, para contemplar a orientação sexual e a identidade de gênero, os quais constituem situações muito mais complexas e são fatores de vulnerabilidade para a saúde.

Pode-se identificar que o desrespeito ao uso do nome social, a discriminação e o preconceito relacionados com a identidade de gênero e com a orientação sexual, somados ao despreparo dos profissionais de saúde para lidar com o público LGBT, são responsáveis pelo absenteísmo e pelo afastamento dessa população aos serviços de saúde. A inabilidade dos profissionais de saúde para lidar com as demandas da população LGBT gera desapontamento com os serviços buscados, descontinuidade de cuidados em saúde e a não procura em outras ocasiões $\mathbf{1 2 , 2 1 , 2 8 , 3 6}$.

A população LGBT vivencia um processo excludente em diversas situações, marcada pela violência em suas variadas formas. Percebendo-se essa realidade, faz-se necessário que o Estado desenvolva políticas públicas eficientes e eficazes a fim minimizar ou acabar com as violações de direitos que essa população sofre. Nesse sentido, diversos estudos concebem o desrespeito ao uso do nome social no SUS e a invisibilidade das demandas e especificidades da população LGBT como formas explícitas de hostilidade a esses sujeitos, levando ao afastamento dos servi-

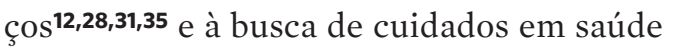
em locais 'alternativos', dentro de instituições não convencionais ${ }^{23}$.

O acesso da população LGBT ao SUS encontra dificuldades de operacionalização uma vez que essa população não segue um padrão heteronormativo; e aquilo que os torna 'diferentes' pode acabar sendo usado contra eles nos serviços de saúde. Por exemplo, a demanda por cuidados específicos e próprios da hormonioterapia e alterações corporais leva a população trans (travestis/ mulheres e homens transexuais) a buscar apenas os serviços ambulatoriais que foram criados com esses fins específicos. Dessa forma, o Estado acaba por incorporar a população LGBT por meio da 
lógica de fluxos assistenciais que contribuem para a exclusão social. Alguns serviços a aborda como infectante, já que estão interligados às demandas de IST e Aids, contrariando a garantia da integralidade da atenção perpassa pelo acesso aos serviços de saúde em todos os níveis de complexidade, e inclui os cuidados básicos com a saúde promovidos nas unidades de saúde 12,21,26,32.

Do mesmo modo, estudos teceram críticas ao modelo biomédico e heteronormativo ${ }^{31}$; discutiram os avanços e barreiras à operacionalização das políticas LGBT ${ }^{17}$; debateram acesso e cuidado integral em saúde à população LGBT ${ }^{27}$, destacaram a necessidade de alterações na formação dos profissionais de saúde, de modo a prepará-los para o atendimento das demandas relacionadas com a orientação sexual e de gênero como estratégia de enfrentamento à discriminação, ao preconceito e ao estigma institucional.

É importante ressaltar que o despreparo para o cuidado em saúde não se restringe aos profissionais que prestam assistência à população LGBT e estende-se a gestores em saúde. Outrossim, o desconhecimento dos objetivos e diretrizes da política LGBT por gestores resulta em desarticulação de ações nos diversos níveis de atenção à saúde, determinando a fragmentação do cuidado ${ }^{32}$.

O desafio da promoção da equidade para a população LGBT deve ser compreendido a partir da perspectiva das suas vulnerabilidades específicas, demandando iniciativas políticas e operacionais que visem à proteção dos direitos humanos e sociais dessas populações. Há um consenso sobre a necessidade do combate à homofobia no SUS e sobre a necessidade de proteção do direito à livre orientação sexual e identidade de gênero, como uma estratégia fundamental e estruturante para a garantia do acesso aos serviços e da qualidade da atenção ${ }^{17,23}$.

Acerca das dificuldades de acesso aos serviços, os artigos apontaram especialmente para fragilidades e obstáculos incutidos no PrTr. Ressaltam que o diagnóstico de transexualismo caracteriza o sujeito como doente, encontra-se amargamente engendrado no sistema e funciona não só como ponte de acesso, mas também como mediador do ingresso do sujeito no $\operatorname{Pr} \operatorname{Tr}^{\mathbf{1 1}, 27}$. A obrigatoriedade diagnóstica, bem como o processo de normatização, "ao mesmo tempo em que gera parâmetros para a garantia de direitos, pode também se prestar a gerar novas demarcações de exclusão"11(96).

Da mesma forma, ao discutir-se o PrTr, salienta-se o pequeno número de hospitais ${ }^{26}$ e ambulatórios habilitados para realização do procedimento no SUS, bem como a sua distribuição geográfica restrita a alguns poucos estados, que acabam por limitar o acesso. Além das barreiras relacionadas com a estrutura física, os autores discutem a necessidade da capacitação dos profissionais no que tange ao acolhimento à população LGBT, à eliminação da discriminação e do estigma nos serviços de saúde.

Também é necessário romper com práticas conservadoras nos espaços públicos e privados que atendem à população LGBT, uma vez que devem ser tratados como cidadãos, implicando assim que também tenham acesso aos serviços e ações de saúde, de forma universal e igualitária. Tais discriminações e estigmatizações se traduzem em barreiras no acesso aos serviços de saúde que podem influenciar na procura por atendimentos de serviços clandestinos, os quais, pela precariedade de forma generalizada, podem oferecer risco e, sobretudo, elevar os padrões de vulnerabilidade, fatos que sinalizam a importância de aumentar o uso de tecnologias leves no cuidado à saúde LGBT ${ }^{25,27}$.

Outro aspecto evidenciado pelos artigos diz respeito à ausência de previsão orçamentária para a execução das ações previstas nos planos, e complementam afirmando que a escassez e até mesmo a ausência de recursos fazem com que as ações sejam difíceis de ser implantadas. Essa restrição indica que, apesar de as políticas e os programas LGBT apresentarem boas perspectivas, a materialização das propostas e ações realizadas ainda é desafiadora ${ }^{\mathbf{7}}$.

Em síntese, as dificuldades de acesso da população LGBT aos serviços de saúde decorrem especialmente do estigma e discriminação 
operados em níveis estrutural, institucional e individual, produzidos por um modelo de assistência à saúde pautado na heteronormatividade. Ademais, o despreparo de gestores e profissionais de saúde para lidar com questões relacionadas com a diversidade sexual e de gênero constitui uma importante barreira tanto para o acesso aos serviços quanto para o cuidado integral à saúde da população LGBT.

\section{Considerações finais}

Ainda que muitas conquistas tenham sido alcançadas quanto à criação de políticas públicas de saúde para população LGBT e que sua visibilidade tenha ampliado, a inserção dessas temáticas nos periódicos da área da Saúde Coletiva tem sido pouco expressiva.

Embora a área da saúde coletiva preveja a interdisciplinaridade na construção do debate, persiste a fragmentação no processo de construção do conhecimento. Os diversos campos de produção de saberes e práticas não romperam com os paradigmas simbólicos e reificantes, perpetuando produções limitadas e estigmatizantes. Tal situação conduz a reflexões alinhadas ao campo da saúde e à premência em instigar a construção de um novo paradigma no processo de ensino nos cursos de saúde, que contemple uma formação acadêmica capaz de discutir a diversidade de gênero como questão social atinente ao processo de cuidado em saúde.
A partir da análise proposta por este estudo emergiram algumas questões que podem suscitar reflexões e produções futuras: o que determina a hierarquização de temas? Por que determinadas temáticas têm maior penetração nessa ou naquela área de concentração da saúde coletiva? Por que, em determinados periódicos, não se discutem questões relacionadas com a população LGBT para além das IST e HIV/Aids? Quais as possíveis barreiras ou impedimentos para a entrada dessa produção científica?

Acredita-se que seja necessário o desenvolvimento de outras pesquisas concernentes à implantação das políticas públicas de saúde LGBT, assim como referente à implementação das ações desenvolvidas até o momento, para a produção de marcadores de saúde que possibilitem a construção de dados epidemiológicos para essa população, e que tais atitudes se desdobrem na repercussão de estratégias para as secretarias municipais de saúde, reais instâncias executoras dos serviços do SUS.

\section{Colaboradores}

Bezerra MVR (0000-0002-6502-6071)*, Moreno CA (0000-0002-1841-2260)*, Prado NMBL (0000-0001-8243-5662)*, Santos AM (0000-0001-9718-1562)* contribuíram igualmente para a elaboração do manuscrito. 


\section{Referências}

1. Atun R, Andrade LO, Almeida G, et al. Health-system reform and universal health coverage in Latin American. Lancet. 2015; 385:1230-47.

2. Paim JS. Reforma Sanitária Brasileira: contribuição para a compreensão e crítica. Salvador; Rio de Janeiro: Edufba; Fiocruz, 2008.

3. Ferrari A. Revisando o passado e construindo o presente: o movimento gay como espaço educativo. Rev Bras de Educação 2004; (25):105-115.

4. Brasil. Ministério da Saúde. Portaria no 2.803 de 19 de novembro de 2013. Redefine e amplia o processo transexualizador no Sistema Único de Saúde. Diário Oficial da União. 20 Nov 2013.

5. Conselho Nacional de Combate à Discriminação. Brasil sem homofobia: programa de combate à violência e à discriminação contra GLBT e promoção da cidadania homossexual. Brasília, DF: CNC; 2004.

6. Mello L, Brito W, Maroja D. Políticas públicas para a população LGBT no Brasil: notas sobre alcances e possibilidades. Cad PAGU. 2012; (39):403-429.

7. Brasil. Programa Nacional de DST e Aids. Plano Nacional de Enfrentamento da Epidemia de AIDS e DSTs entre Gays, Homens que fazem sexo com homens (HSH) e Travestis. Brasília, DF: MS; 2007.

8. Brasil. Ministério da Saúde. Plano Nacional de Promoção da Cidadania e Direitos Humanos de LGBT. Brasília, DF: MS; 2009.

9. Brasil. Ministério da Saúde. Portaria n ${ }^{\circ} 2.836$ de 1 de dezembro de 2011. Política Nacional de Saúde Integral de Lésbicas, Gays, Bissexuais, Travestis e Transexuais. Diário Oficial da União. 2 Dez 1999.

10. Brasil. Secretaria de Atenção à Saúde. Cadernos de Atenção Básica no 26: Saúde Sexual e Saúde Reprodutiva. Brasília, DF: MS; 2010.
11. Lionço T. Atenção integral à saúde e diversidade sexual no processo transexualizador do SUS: avanços, impasses, desafios. Physis. 2009; 19(1):43-63.

12. Silva LKM, Silva ALMA, Coelho AA, et al. Uso do nome social no Sistema Único de Saúde: elementos para o debate sobre a assistência prestada a travestis e transexuais. Physis. 2017; 27(3):835-846.

13. Brignol S, Dourado I, Amorim LD, et al. Vulnerabilidade no contexto da infecção por HIV e sífilis numa população de homens que fazem sexo com homens (HSH) no Município de Salvador, Bahia, Brasil. Cad. Saúde Pública. 2015; 31(5):1-14.

14. Magno L, Dourado I, Silva LA, et al. Factors associated with self-reported discrimination against men who have sex with men in Brazil. Rev Saude Publica. 2017; (51):102-113.

15. Pinto TP, Teixeira FB, Barros CRS, et al. Silicone líquido industrial para transformar o corpo: prevalência e fatores associados ao seu uso entre travestis e mulheres transexuais em São Paulo, Brasil. Cad. Saúde Pública. 2017; 33(7):1-13.

16. Rocha RMG, Pereira DL, Dias TM. O contexto do uso de drogas entre travestis profissionais do sexo. Saúde Soc. 2013; 22(2):554-565.

17. Albuquerque GA, Garcia CL, Alves MJH, et al. Homossexualidade e o direito à saúde: um desafio para as políticas públicas de saúde no Brasil. Saúde debate. 2013 ; 37(98):516-524.

18. Almeida G. Argumentos em torno da possibilidade de infecção por DST e Aids entre mulheres que se autodefinem como lésbicas. Physis. 2009; 19(2):301-331.

19. Angonese M, Lago MCS. Direitos e saúde reprodutiva para a população de travestis e transexuais: abjeção e esterilidade simbólica. Saúde Soc. 2017; 26(1):256270. 
20. Motta JIJ. Sexualidades e políticas públicas: uma abordagem queer para tempos de crise democrática. Saúde debate. 2016; 40(esp):73-86.

21. Barbosa RM, Facchini R. Acesso a cuidados relativos à saúde sexual entre mulheres que fazem sexo com mulheres em São Paulo, Brasil. Cad. Saúde Pública 2009: 25(supl2):S291-S300.

22. Freire EC, Araújo FCA, Souza AC, et al. A clínica em movimento na saúde de TTTS: caminho para materialização do SUS entre travestis, transsexuais e transgêneros. Saúde debate. 2013; 37(98):477-484.

23. Souza MHT, Signorelli MC, Coviello DM, et al. Itinerários terapêuticos de travestis da região central do Rio Grande do Sul, Brasil. Ciênc. Saúde Colet. 2014; 19(7):2277-2286.

24. Rocon PC, Rodrigues A, Zamboni J, et al. Dificuldades vividas por pessoas trans no acesso ao Sistema Único de Saúde. Ciênc. Saúde Colet. 2016; 21(8):25172525.

25. Ferreira BO, Nascimento EF, Pedrosa JIS, et al. Vivências de travestis no acesso ao SUS. Physis. 2017; 27(4):1023-1038.

26. Popadiuk GS, Oliveira DC, Signorelli MC. A Política Nacional de Saúde Integral de Lésbicas, Gays, Bissexuais e Transgêneros (LGBT) e o acesso ao Processo Transexualizador no Sistema Único de Saúde (SUS): avanços e desafios. Ciênc. Saúde Colet. 2017; 22(5):1509-1520.

27. Rocon PC, Sodré F, Zamboni J, et al. O que esperam pessoas trans do Sistema Único de Saúde Interface - Comunic, Saude, Educ. 2018; 22(64):43-53.

28. Sousa D, Iriart J. "Viver dignamente": necessidades e demandas de saúde de homens trans em Salvador, Bahia, Brasil. Cad. Saúde Pública. 2018; 34(10):e00036318.

29. Ramos S, Carrara S. A Constituição da Problemáti- ca da Violência contra Homossexuais: a Articulação entre Ativismo e Academia na Elaboração de Políticas Públicas. Physis. 2006; 16(2):185-205.

30. Lionço T. Que Direito à Saúde para a População GLBT? Considerando Direitos Humanos, Sexuais e Reprodutivos em Busca da Integralidade e da Eqüidade. Saúde Soc. 2008; 17(2):11-21.

31. Valadão RC, Gomes R. A homossexualidade feminina no campo da saúde: da invisibilidade à violência. Physis. 2011; 21(4):1451-1467.

32. Gomes SM, Sousa LMP, Vasconcelos TM, et al. O SUS fora do armário: concepções de gestores municipais de saúde sobre a população LGBT. Saúde Soc. 2018; 27(4):1120-1133.

33. Zago LF, Santos LHS. Os limites do conceito de empoderamento na prevenção ao HIV/Aids entre jovens gays e bissexuais no Brasil. Physis. 2013; 23(3):681701.

34. Redoschi BRL, Zucchi EM, Barros CRS, et al. Uso rotineiro do teste anti-HIV entre homens que fazem sexo com homens: do risco à prevenção. Cad. Saúde Publica. 2017; 33(4): 1-22.

35. Silva CG, Paiva V, Parker R. Juventude religiosa e homossexualidade: desafios para a promoção da saúde e de direitos sexuais. Interface - Comunic, Saude, Educ. 2013; 17(44):103-17.

36. Taquette SR, Rodrigues AO. Experiências homossexuais de adolescentes: considerações para o atendimento em saúde. Interface - Comunic, Saude, Educ. 2015; 19(55):1181-91.

37. Facchini R. "Sopa de Letrinhas?” Movimento homossexual e produção de identidades coletivas nos anos 90: um estudo a partir da cidade de São Paulo. [dissertação]. Campinas: Departamento de Antropologia Social, Instituto de Filosofia e Ciências Humanas, Universidade Estadual de Campinas; 2002. 
38. Brasil. Ministério da Saúde. Carta dos direitos dos usuários da saúde. Brasília, DF: MS; 2007.

39. Brasil. Plano Integrado de enfrentamento da feminização da Epidemia de Aids e outras DST. Brasília, DF: MS; 2007.

40. Brasil. Portaria $n^{\circ} 457$ de 19 de agosto de 2008. Institui o processo transexualizador no Sistema Único de Saúde. Brasília, DF: Ministério da Saúde; 2008.
41. Albuquerque GA, Parente JS, Belém JM, et al. Violência psicológica em lésbicas, gays, bissexuais, travestis e transexuais no interior do Ceará, Brasil. Saúde debate. 2016; 40(109):100-111.

Recebido em 02/06/2019

Aprovado em 16/01/2020

Conflito de interesses: inexistente

Suporte financeiro: não houve 